\title{
Severe Hypocalcaemia and Hypomagnesaemia Secondary to Omeprazole
}

Claire Min-Li Teo, Timothy Peng Lim Quek, Shaikh A. K.K. Abdul Shakoor

Department of Endocrinology, Tan Tock Seng Hospital, Republic of Singapore

\section{Introduction}

Proton pump inhibitors (PPIs) are commonly prescribed drugs. We report a case of severe hypocalcaemia secondary to omeprazole induced hypomagnesaemia.

\section{Case Report}

An 85 year old Chinese man with stage 4 chronic kidney disease was admitted to our hospital in Jun 2014 with vomiting, loss of appetite and generalized weakness. On admission, he was severely hypocalcaemic (adjusted calcium $1.37 \mathrm{mmol} / \mathrm{L}$ [RI: 2.15-2.58]) and hypomagnesaemic (Mg 0.1 mmol/L [RI: 0.71.0]). Serum phosphate and 25-OH Vitamin D levels were normal. Intact PTH done after normalisation of serum magnesium showed secondary hyperparathyroidism (intact PTH $61.0 \mathrm{pmol} / \mathrm{L}$ [RI: 0.8-6.8]).

An inpatient Endocrinology referral was made for persistent hypocalcaemia despite aggressive intravenous replacement over 3-4 days. Review of his medical records revealed initiation of omeprazole in 2010 for peptic ulcer prophylaxis when he was prescribed short course prednisolone for a gout flare. Omeprazole was continued after prednisolone cessation. Severe hypomagnesaemia and hypocalcaemia was also noted during 2 previous admissions to another hospital in Nov 2013 and Jun 2014. Calcium levels were normal between hospitalisations but magnesium levels were not rechecked.

In view of the possibility of omeprazole induced hypomagnesaemia and hypocalcaemia, omeprazole was stopped and changed to famotidine. With electrolyte replacement and a short course of calcitriol, serum calcium normalised within 4 days and remained normal at follow-up with calcium carbonate $1.25 \mathrm{~g}$ bd (as a phosphate binder) and no magnesium supplementation.

\section{Trend of Calcium and Magnesium levels}

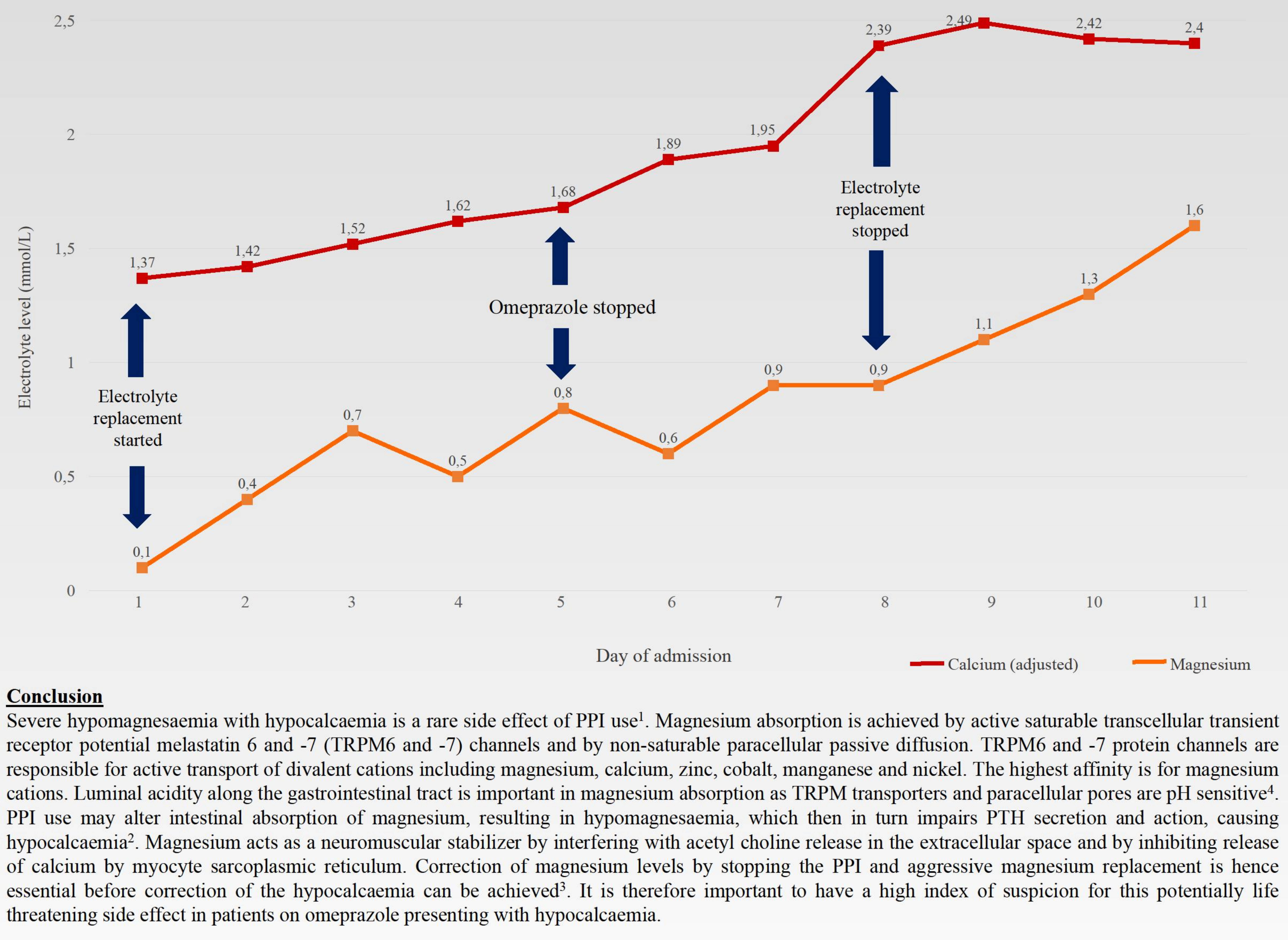

$\frac{\text { References }}{\text { Park Chan }}$

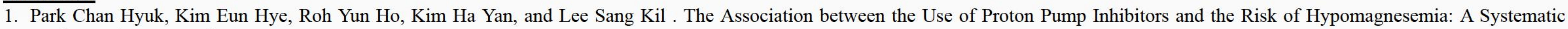
Review and Meta-Analysis. PLoS One. 2014; 9(11): e112558.

2. Cundy T, Dissanayake A. Severe hypomagnesaemia in long-term users of proton-pump inhibitors. Clin Endocrinol (Oxf) $2008 ; 69(2): 338-41$.

3. Shabajee N, Lamb EJ, Sturgess I, Sumathipala RW. Omeprazole and refractory hypomagnesaemia. BMJ. 2008 Jul 10;337:a425. doi:10.1136/bmj.39505.738981.BE. 